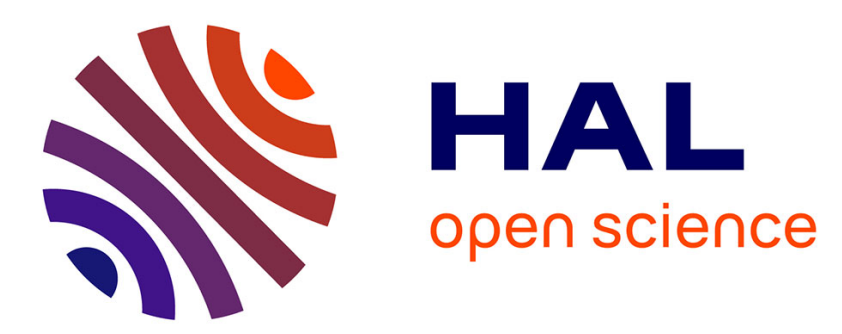

\title{
Improving Moments-based Visual Servoing with Tunable Visual Features
}

\author{
Manikandan Bakthavatchalam, Omar Tahri, François Chaumette
}

\section{To cite this version:}

Manikandan Bakthavatchalam, Omar Tahri, François Chaumette. Improving Moments-based Visual Servoing with Tunable Visual Features. IEEE Int. Conf. on Robotics and Automation, ICRA'14, Jun 2014, Hong-Kong, Hong Kong SAR China. hal-00949163

\section{HAL Id: hal-00949163 \\ https://hal.inria.fr/hal-00949163}

Submitted on 19 Feb 2014

HAL is a multi-disciplinary open access archive for the deposit and dissemination of scientific research documents, whether they are published or not. The documents may come from teaching and research institutions in France or abroad, or from public or private research centers.
L'archive ouverte pluridisciplinaire HAL, est destinée au dépôt et à la diffusion de documents scientifiques de niveau recherche, publiés ou non, émanant des établissements d'enseignement et de recherche français ou étrangers, des laboratoires publics ou privés. 


\title{
Improving Moments-based Visual Servoing with Tunable Visual Features
}

\author{
Manikandan Bakthavatchalam, Omar Tahri, François Chaumette
}

\begin{abstract}
In this paper, we introduce the concept of tunable visual features for moments based visual servoing schemes. The main contribution of this work is the introduction of tunable shift points along with some effective methods to tune them. We propose two different metrics: the first metric ensures optimal response of the control to errors in the image space and the second metric ensures orthogonality between the interaction matrix components (vectors) related to the control of $x$ and $y$ rotational motions. With the proposed method, it is possible to design moment invariants-based visual features whose interaction matrix is always non-singular for any desired pose (parallel or non-parallel). Thus, this work makes a significant contribution to the difficult problem of controlling the rotational motions around the $x$ and $y$ axes, when all the 6dof are involved. Two case studies are presented to demonstrate the validity of the proposed ideas. Results from each case are then used to design a moment invariants-based visual feature. This visual feature is used for visual servoing with a symmetrical object using binary moments and a freeform planar target using photometric moments.
\end{abstract}

\section{INTRODUCTION}

Visual servoing (VS) is the technique of controlling the motions of a robotic system using information from a visual sensor such as a camera. In visual servoing, there are two closely linked problem themes that are subjects of active research. The first is the design of visual features most pertinent to the robotic task to be realized. The second is to devise a control scheme with the chosen visual features such that the desired characteristics are obtained during the visual servo. The relationship between the variation in the selected visual features to the movement of the visual sensor is modeled by the well-known equation [1].

$$
\dot{\mathbf{s}}(t)=\mathbf{L}_{s} \mathbf{v}_{c}
$$

where $\mathbf{v}_{c}$ is the cartesian velocity expressed in the camera frame and $\mathbf{L}_{s}$ is the interaction matrix of the chosen visual features. Initially, the feature set $\mathbf{s}(t)$ was related to planar geometric (points, straight lines, circles) or volumetric primitives (spheres, cylinders). These methods require that the scene observed by the visual sensor can be modelled by such simple primitives, thus naturally limiting their domain of applicability. Further, feature tracking and/or matching algorithms need to be integrated into the control loop which consumes bandwidth that can be otherwise used by the control. This was identified long back as a stumbling block for the expansion of VS [2]. The recent vision paper [3] highlighted challenges that have to be overcome in order to make visual servoing methods practically useful in real-world applications.
Position-based visual servoing (PBVS) [1] acted upon the cartesian errors which produced satisfactory servoing characteristics. However, a robust pose estimation and knowledge of the 3D target model are essential. A blend of IBVS and PBVS known as the 2.5D method [4], then entered the arena but a partial pose estimation was still required.

As a means to overcome the aforementioned issues, visual servoing techniques based on the luminance [5] and information-theoretic approaches like [6] were developed. While these methods do not require tracking or matching, they are non-linear in nature and so their convergence domain is limited.

Image moments represent the essential properties of the image signal and were adopted for visual servoing in several works [7]-[10]. More recently, photometric image moments [11] were introduced as visual features for visual servoing. In this work, free-form planar targets were used without any visual tracking or feature matching. Also, it clearly pointed out that a non-optimal choice of visual features resulted in unsatisfactory behaviour during 6dof servo. As is shown in this paper, the method we propose is also applicable to photometric moments.

In visual servoing, it is well-known that the simultaneous control of the translations together with rotational motions perpendicular to the optic axis is a non-trivial task. In [12], the authors proposed using directional Gaussian kernels to control the planar translations and the discrete fourier transform (DFT) to control the translation along and rotation around the optic axis. The control of the other critical degrees of freedom was completely ignored. The work [10] showed that image moments are well suited for a SCARA type task, restricted to only 4-dof. The problem was back in focus, thanks to [13] which proposed using lower order moments computed with shift points along the major and minor orientation axes. But this choice is not optimal in all cases. We will present new tunable shift points that can be selected optimally.

\section{Customizable Visual Features}

\section{A. Preliminaries and related work}

The main idea of this paper is the design of customizable visual features that finds immediate applications in image moments-based visual servoing. Let us begin this paper by introducing some basic concepts and closely related works which will help in understanding this paper. In general, 
the moments of an image are defined as

$$
m_{p q}=\iint_{\pi} x^{p} y^{q} I(x, y) \mathrm{d} x \mathrm{~d} y
$$

where $I(x, y)$ is the image intensity function, $p+q$ denotes the order of the moment and $\pi$ denotes the image plane. Clearly, if $I(x, y) \in\{0,1\} \forall(x, y)$, then the image is binary and (2) represents binary moments. More recently, photometric image moments [11] were introduced in visual servoing, where intensities in all the image plane was taken into account.

Many image moments based visual servoing methods [8], [13], [11] often employ a set of visual features $\mathbf{s}$ as follows:

$$
\mathbf{s}=\left(x_{n}, y_{n}, a_{n}, \phi_{1}, \phi_{2}, \alpha\right)
$$

where $\left\{x_{n}=x_{g} \cdot a_{n} \quad y_{n}=y_{g} \cdot a_{n} \quad a_{n}=z^{*} \cdot \sqrt{\frac{a^{*}}{a}}\right.$

$x_{n}$ and $y_{n}$ are the coordinates of the normalized centre of gravity and $a_{n}$ is the normalized area. $\alpha=\frac{1}{2} \arctan \left(\frac{2 \mu_{11}}{\mu_{02}+\mu_{20}}\right)$ represents the orientation of the target. These 4 features have been used in several works including [8], [13] and [11] and result in a decoupled interaction matrix when the desired camera and target planes are parallel. $\phi_{1}$ and $\phi_{2}$ are visual features that are typically used to control the rotational motions around the $x$ and $y$ camera axes. These features are based on moment invariants built from the centered moments of the image. The centered moments are invariant to planar translations and are defined as

$$
\mu_{p q}=\iint\left(x-x_{g}\right)^{p}\left(y-y_{g}\right)^{q} \mathrm{~d} x \mathrm{~d} y
$$

The idea of shifted moments was first proposed in [13], whereby the image moments were computed along specially chosen shift points. These moments were termed shifted moments and are given by

$$
\tilde{\mu}_{p q}=\iint\left(x-x_{g}+\tilde{x}\right)^{p}\left(y-y_{g}+\tilde{y}\right)^{q} I(x, y) \mathrm{d} x \mathrm{~d} y
$$

where $\tilde{\mu}_{p q}$ are the shifted centered moments of order $p+q$. In [13], the following shift points were proposed: Shift point $\mathrm{p}_{1}$

$$
\begin{gathered}
\tilde{x}=\left(\mu_{20}+\mu_{02}\right)^{\frac{1}{4}} \cos (\alpha) \\
\tilde{y}=\left(\mu_{20}+\mu_{02}\right)^{\frac{1}{4}} \sin (\alpha) \\
\text { Shift point } \mathrm{p}_{2} \\
\tilde{x}=\left(\mu_{20}+\mu_{02}\right)^{\frac{1}{4}} \cos \left(\alpha+\frac{\pi}{2}\right) \\
\tilde{y}=\left(\mu_{20}+\mu_{02}\right)^{\frac{1}{4}} \sin \left(\alpha+\frac{\pi}{2}\right)
\end{gathered}
$$

where $\alpha$ is the orientation as in (3). We can see that the shift points were computed with the orientation axes shifted by 0 and $\pi / 2$. However, this is not always the best choice. In fact, a bad choice might affect the stability of the system, cause singularities and might lead to failure of the control. In this paper, we posit that the shift points need not

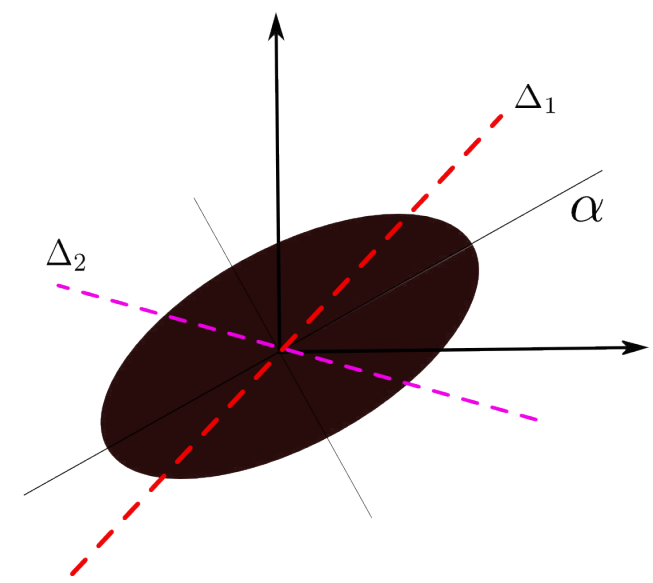

Fig. 1: Illustration for shifted moments. $\alpha$ is along the major axis of the orientation ellipse. $\Delta_{1}$ and $\Delta_{2}$ are shifts in orientation that define the actual shift points

always be chosen in this manner. We present a generalized version of the shift points called tunable shift points that can be applied in moments-based visual servoing. Visual features customized to satisfy specific criteria can be built from these shift points. Later, we will show that the shifted moments proposed in [13] are a specific manifestation of our generalized case.

The tunable shift points that we propose can be defined as:

$$
\begin{aligned}
& \text { Tunable shift point } \mathrm{p}_{1} \\
& x_{s 1}=\sqrt{m_{00}} \cos \left(\alpha+\Delta_{1}\right) \\
& y_{s 1}=\sqrt{m_{00}} \sin \left(\alpha+\Delta_{1}\right) \\
& \text { Tunable shift point } \mathrm{p}_{2} \\
& x_{s 2}=\sqrt{m_{00}} \cos \left(\alpha+\Delta_{2}\right) \\
& y_{s 2}=\sqrt{m_{00}} \sin \left(\alpha+\Delta_{2}\right)
\end{aligned}
$$

First, we note that, unlike in [13], instead of the second order moments, $m_{00}$ has been used. Such choices, coupled with appropriate visual features, has positive implications for the stability of the system. A formal proof of stability is beyond the scope of this paper. Indeed, it is advisable in general to stick to the lowest order moments possible since higher order moments are sensitive to noise [14]. Next, we present our methods to select optimal shift points based on specific measures obtained from the interaction matrix. Image moments based on these shift points can then be computed and used for optimal visual servoing.

\section{B. Selection of $\Delta_{1}$}

As already said, a challenging aspect in visual servoing is the simultaneous control of the rotational motions around the $x$ and $y$ camera axes, when all 6dof of the robot have to be controlled. The interaction matrix for a pair of visual 
features built from shifted moments has the general form ${ }^{1}$ :

$$
\mathbf{L}_{i}=\left[\begin{array}{llllll}
L_{\mathrm{p}_{1}}^{v_{x}} & L_{\mathrm{p}_{1}}^{v_{y}} & L_{\mathrm{p}_{1}}^{v_{z}} & L_{\mathrm{p}_{1}}^{\omega_{x}} & L_{\mathrm{p}_{1}}^{\omega_{y}} & L_{\mathrm{p}_{1}}^{\omega_{z}} \\
L_{\mathrm{p}_{2}}^{v_{x}} & L_{\mathrm{p}_{2}}^{v_{y}} & L_{\mathrm{p}_{2}}^{v_{z}} & L_{\mathrm{p}_{2}}^{\omega_{x}} & L_{\mathrm{p}_{2}}^{\omega_{y}} & L_{\mathrm{p}_{2}}^{\omega_{z}}
\end{array}\right]
$$

Each entry in the interaction matrix of a visual feature represents the responsiveness of that visual feature $i$ to the task errors (in image space in IBVS). Delving slightly deeper and observing the invariant visual feature that we propose, its interaction matrix is a function of the moments computed with respect to tunable shift points (such as $\mathrm{p}_{1}$ and $\mathrm{p}_{2}$ ). By selecting an optimal $\Delta_{1}$ connected to the first shift point, we can obtain an interaction matrix with optimal responsiveness with respect to $\omega_{x}$ and $\omega_{y}$. This can be formally stated as the following optimization problem:

$$
\Delta_{1}^{*}=\max _{\Delta_{1}} \mathcal{F}_{1}\left(\Delta_{1}\right)
$$

where

$$
\mathcal{F}_{1}\left(\Delta_{1}\right)=\left\|\mathbf{L}_{\mathrm{p}_{1}}^{\omega}\right\|=\left\|\left[\begin{array}{ll}
L_{\mathrm{p}_{1}}^{\omega_{x}} & L_{\mathrm{p}_{1}}^{\omega_{y}}
\end{array}\right]\right\|
$$

where the operator $\|$.$\| stands for l_{2}$ norm. The solution of this optimization will ensure that the control law (using as visual feature $i_{3}$ in (20) or others) acts optimally on the corresponding errors in the image space. Once the optimal value of $\Delta_{1}$ is obtained, first shift point can be held constant at this value $\Delta_{1}^{*}$ and the second shift point should be selected.

\section{Selection of $\Delta_{2}$}

The $2 \times 2$ partial interaction matrix $\mathbf{L}_{\omega_{x, y}}$ related to the $x$ and $y$ rotational motions can be observed from Eq.(10).

$$
\mathbf{L}_{\omega_{x, y}}=\left[\begin{array}{ll}
L_{\mathrm{p}_{1}}^{\omega_{x}} & L_{\mathrm{p}_{1}}^{\omega_{y}} \\
L_{\mathrm{p}_{2}}^{\omega_{x}} & L_{\mathrm{p}_{2}}^{\omega_{y}}
\end{array}\right]=\left[\begin{array}{l}
\mathbf{L}_{\mathrm{p}_{1}} \\
\mathbf{L}_{\mathrm{p}_{2}}
\end{array}\right]
$$

The selection of $\Delta_{2}$ should be such that the simultaneous control of both the rotations is optimal. We propose to choose $\Delta_{2}$ such that the factors responsible for the $x$ and $y$ rotational velocities are perpendicular to each other. That is, $\Delta_{2}^{*}$ should belong to the solution set that satisfies this orthogonality of the vectors $\mathbf{L}_{\mathrm{p}_{2}}$ and $\mathbf{L}_{\mathrm{p}_{1}}$.

$$
\Delta_{2}^{*} \mid \mathcal{F}_{2}\left(\Delta_{2}\right)=0
$$

where

$$
\mathcal{F}_{2}\left(\Delta_{2}\right)=\kappa_{d n}\left(\mathbf{L}_{\omega_{x, y}}\right)=\frac{\mathbf{L}_{\mathrm{p}_{1}}^{\top} \cdot \mathbf{L}_{\mathrm{p}_{2}}}{\left\|\mathbf{L}_{\mathrm{p}_{2}}\right\|}
$$

where $\kappa_{d n}$ is a function of the partial interaction matrix and is the angle between the two vectors $\mathbf{L}_{\mathrm{p}_{1}}$ and $\mathbf{L}_{\mathrm{p}_{2}}$. Clearly, these two vectors are a function of the respective visual feature chosen to control the $x$ and $y$ rotations. With this optimization, these vectors are optimized in such a way that their directions are perpendicular to each other. While $\Delta_{1}$ is usually uniquely found, there could be several choices for $\Delta_{2}$ all of which satisfy the orthogonality criterion proposed in (15). (In practice, when using $i_{3}$ in (20) as a visual feature, multiple solutions were usually found). In this case,

\footnotetext{
${ }^{1}$ In each entry of $\mathbf{L}$, the subscript signifies the shift point $p_{i}$ at which the invariant is evaluated. The superscript $v$ refers to the translational velocity and $\omega$ to the rotational velocity component of the camera, in either of $\mathrm{x}, \mathrm{y}$ or $\mathrm{z}$ axes
}

one among them, which is greater and farther from the optimal value of $\Delta_{1}$ can be selected. However, this is not the only possible choice. Depending upon the problem at hand, one can also employ other suitable objective functions. For example, $\Delta_{2}$ can be easily tied to the stability of the system. The condition number of the interaction matrix has been used in [15] and measures such as resolvability [16] and motion perceptibility [17] are based on the interaction matrix (referred to as image jacobian in the above works). For instance, we can seek the best conditioning of the partial interaction matrix $\mathbf{L}_{\omega_{x, y}}$. That is,

$$
\Delta_{2}^{*}=\max _{\Delta_{2}} \mathcal{F}_{\text {stab }}\left(\Delta_{2}\right)
$$

where

$$
\mathcal{F}_{\text {stab }}\left(\Delta_{2}\right)=\kappa\left(\mathbf{L}_{\mathbf{s}}\right)=\frac{1}{\operatorname{cond}\left(\mathbf{L}_{\omega_{x, y}}\right)}
$$

where $\kappa$ is defined as the inverse of the condition number of $\mathbf{L}_{\omega_{x, y}}$. In theory, $\kappa$ can be any metric that is a reliable indicator of the stability of the system. Further, if moment invariants are selected and the desired configuration is such that the image and the object planes are parallel, then the interaction matrix will exhibit a decoupled structure as follows:

$$
\mathbf{L}_{i}^{*}=\left[\begin{array}{cccccc}
0 & 0 & 0 & L_{\mathrm{p}_{1}}^{\omega_{x}} & L_{\mathrm{p}_{1}}^{\omega_{y}} & 0 \\
0 & 0 & 0 & L_{\mathrm{p}_{2}}^{\omega_{x}} & L_{\mathrm{p}_{2}}^{\omega_{y}} & 0
\end{array}\right]
$$

But the method is not limited to parallel configurations. For the general case like in (10), we can choose an appropriate objective to be minimized.

$$
\mathcal{F}_{3}\left(\Delta_{2}\right)=\kappa\left(\mathbf{L}_{\mathbf{s}}\right)=\frac{1}{\operatorname{cond}\left(\mathbf{L}_{\mathbf{s}}\right)}
$$

In fact, we performed a visual servoing using both the objective functions, (15) and (19) for the non-parallel configuration (in which the camera and target planes are not parallel to each other). The servo converged with no issues. It has to be noted that (19) takes the conditioning of the entire system into account which is useful from the stability point-of-view. Also, in [8], a method based on virtual rotations has been proposed for use in non-parallel configurations. This can also be equally used. We have thus proposed some metrics for visual feature selection that are tied to responsiveness with respect to $\omega_{x}$ and $\omega_{y}$, orthogonality in components of partial interaction matrix and to the conditioning of the system.

\section{CASE STUdIES}

Two case studies are presented: the first one with a simple symmetrical target using binary moments and the second with a full grayscale texture using photometric moments. A calibrated camera ${ }^{2}$ was assumed and images of size $640 \times 480$ were used. The camera simulator was developed by interfacing to the ViSP software library [18]. The first configuration was chosen such that the image and the target planes are parallel at a depth of $1.0 \mathrm{~m}$ above the target. That is ${ }^{c *} \mathbf{M}_{o}^{p}=\left[0.0,0.0,1 \mathrm{~m}, 0.0^{\circ}, 0.0^{\circ}, 0.0^{\circ}\right]$. As for the non-parallel configuration the following pose ${ }^{c *} \mathbf{M}_{o}=$

$$
{ }^{2}(p x=1200, p y=900, u 0=320, v 0=240)
$$


$\left[20.0 \mathrm{~cm},-20.0 \mathrm{~cm}, 280 \mathrm{~cm},-20.0^{\circ}, 20.0^{\circ}, 20.0^{\circ}\right]$ was used. Typically, there is an extremely large displacement in depth. In this work, we used the visual feature $i_{3}$ :

$$
i_{3}=\frac{I_{s 3}}{I_{s 2}}
$$

where

$$
\begin{aligned}
& I_{s 2}=-\tilde{\mu}_{30} \tilde{\mu}_{12}+\tilde{\mu}_{21}^{2}-\tilde{\mu}_{03} \tilde{\mu}_{21}+\tilde{\mu}_{12}^{2} \\
& I_{s 3}=3 \tilde{\mu}_{30} \tilde{\mu}_{12}+\tilde{\mu}_{30}^{2}+3 \tilde{\mu}_{03} \tilde{\mu}_{21}+\tilde{\mu}_{12}^{2}
\end{aligned}
$$

The ratio $i_{3}$ is made up of moment polynomials that scale equally with depth and was proposed in [13]. Clearly, they are built from shifted moments of up to third order(see (20) and (5)). But the method can also be applied to any other moment invariants-based visual feature. We recall to the reader that the visual features are now calculated using the tunable shift points (8),(9) introduced in this paper. Further, in the case studies that follow, the metrics (11) and (15) was used for $\Delta_{1}$ for $\Delta_{2}$ selection respectively.

\section{A. Case I}

For the symmetric case a simple white rectangle was used as the target. This is a symmetric object and binary moments were used in this case. This serves as a validation step before moving on to the photometric moments.

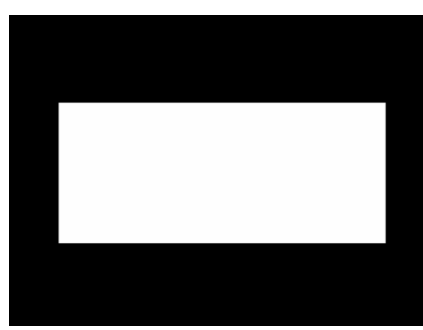

(a)

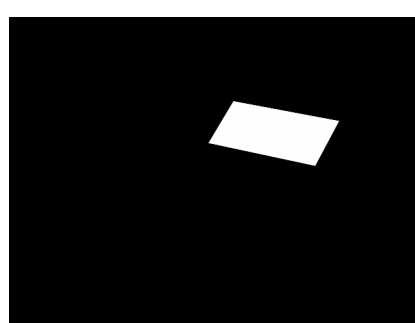

(b)
Fig. 2: Camera views of symmetrical object at parallel and non-parallel camera poses

In the case of the parallel configuration, using our method, we found that $\Delta_{1}^{*}=180^{\circ}$ and $\Delta_{2}^{*}=149^{\circ}$. Indeed from Fig.3a, we can infer that the variation of $\mathcal{F}_{1}$ presents a symmetry and the optimal value has been predicted at the centre of this symmetry. As for $\Delta_{2}$, there are several possible candidate directions that might ensure the orthognality condition. We chose the direction that was farthest from the optimal $\Delta_{1}$. For the non-parallel configuration, we arrived at $\Delta_{1}^{*}=180^{\circ}$ and $\Delta_{2}^{*}=311^{\circ}$ respectively.

\section{B. Case II}

For this case, we used the graffiti image (see Fig.4). The grayscale image can be considered to have a random intensity distribution. One objective of this case study was to show that the method can be applied to photometric image moments introduced recently in [11].

At the parallel desired configuration, using the metrics we propose, the optimal values of $\Delta_{1}^{*}=3^{\circ}$ and $\Delta_{2}^{*}=102^{\circ}$

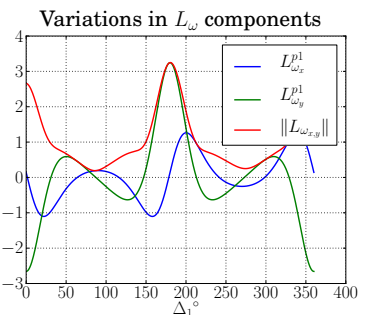

(a)

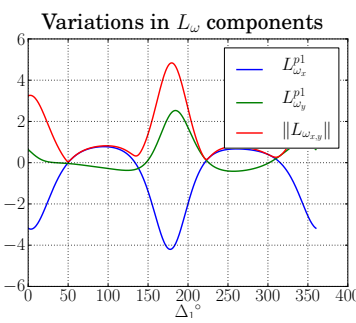

(c)

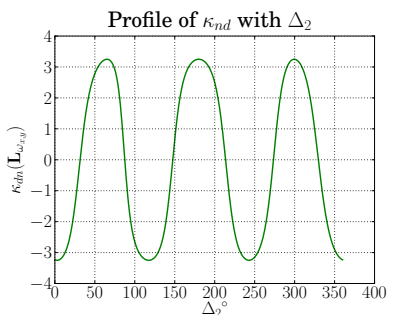

(b)

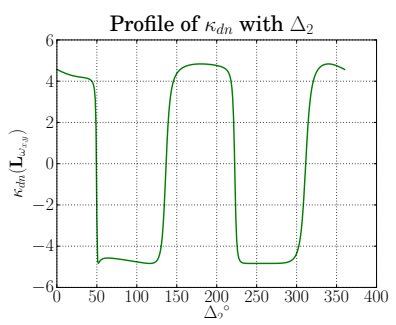

(d)
Fig. 3: Variations in interaction matrix for symmetric target (a) $\mathbf{L}_{\mathrm{p} 1}$ variations with respect to $\Delta_{1}$ and (b) Orthogonality with respect to $\Delta_{2}$ for parallel camera configuration, (c) $\mathbf{L}_{\mathrm{p} 1}$ variations with respect to $\Delta_{1}$ and (d) Orthogonality with respect to $\Delta_{2}$ for non-parallel camera configuration

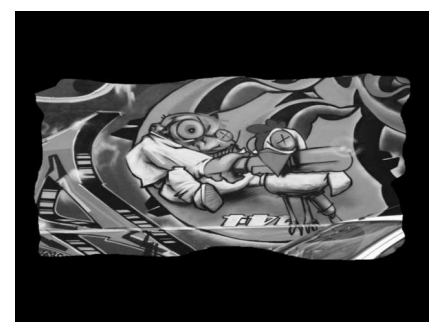

(a)

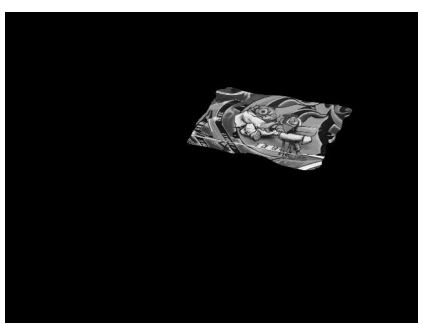

(b)
Fig. 4: Camera views of graffiti texture at parallel and nonparallel desired configurations

are chosen. As evidenced by Fig.5a, the changes in the interaction matrix with respect to $\Delta_{1}$ is not periodic or symmetrical. With this method, we obtained $\Delta_{1}^{*}=176^{\circ}$ and $\Delta_{2}^{*}=300^{\circ}$ for the non parallel configuration.

\section{Visual Servoing Simulation Results}

We performed a set of visual servoing simulations with shift points selected as shown in Sec.III. As mentioned earlier, the below set of visual features were used:

$$
\mathbf{s}=\left(x_{n}, y_{n}, a_{n}, i_{3 \mathrm{p} 1}, i_{3 \mathrm{p} 2}, \alpha\right)
$$

where feature $i_{3}$ is used in controlling the $x$ and $y$ rotations and use the values of the shift point with $\Delta_{1}$ and $\Delta_{2}$ obtained with metrics (11) and (15).The other four features are frequently used in several works including [8], [11] and [13].

We assume that the camera is mounted on the endeffector in a typical eye-in-hand configuration. The camera 


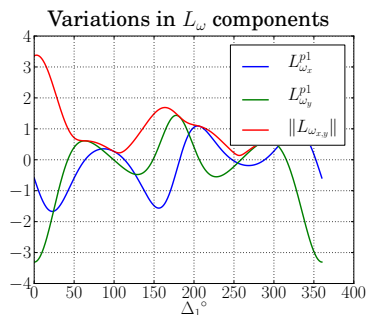

(a)

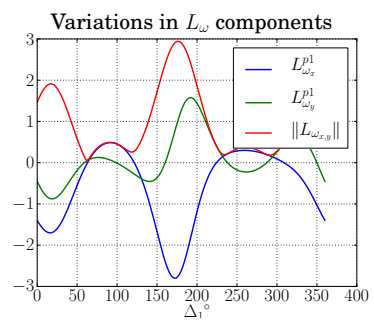

(c)

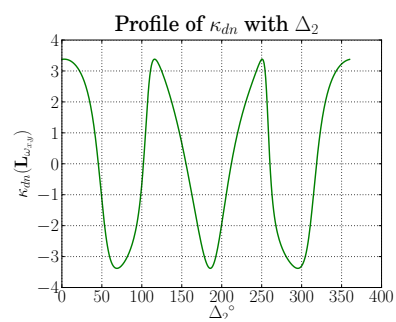

(b)

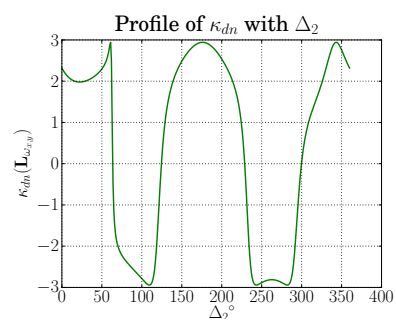

(d)
Fig. 5: Features design through shift points for photometric target (a) $\mathbf{L}_{\mathrm{p} 1}$ variations with respect to $\Delta_{1}$ and (b) Orthogonality with respect to $\Delta_{2}$ for parallel camera configuration, (c) $\mathbf{L}_{\mathrm{p} 1}$ variations with respect to $\Delta_{1}$ and (d) Orthogonality with respect to $\Delta_{2}$ for non-parallel camera configuration

velocities are then given by the classic control law:

$$
\mathbf{v}_{c}=-\lambda \hat{\mathbf{L}}_{\mathbf{s}}^{-1}\left(\mathbf{s}-\mathbf{s}^{*}\right)
$$

A true value of $\mathbf{L}_{\mathbf{s}}$ is difficult to achieve in practice and an estimation of this matrix denoted $\hat{\mathbf{L}}_{\mathbf{s}}$ is normally used. Different choices are possible for its evaluation as explained in [1]. In this work, we use the interaction matrix computed at the desired pose, that is $\mathbf{L}_{\mathbf{s}}=\mathbf{L}_{\mathbf{s} *}$. A constant gain of $\lambda=$ 0.6 was used in all the experiments. The desired pose was chosen such that it was parallel to the target plane. Typically, ${ }^{c *} \mathbf{M}_{o}^{p}=\left[0.0,0.0,1 m, 0.0^{\circ}, 0.0^{\circ}, 0.0^{\circ}\right]$. The initial pose was selected such that a huge displacement is required for the realisation of the task. In this paper, the following initial pose ${ }^{c} \mathbf{M}_{o}^{i}=\left[20.0 \mathrm{~cm},-20.0 \mathrm{~cm}, 280 \mathrm{~cm},-20.0^{\circ}, 20.0^{\circ}, 20.0^{\circ}\right](=$ ${ }^{c *} \mathbf{M}_{o}$ ) was used. Then, for testing the visual servoing with a non-parallel desired configuration, the above poses are just interchanged. Results from both these scenarios are presented in Fig.6 for the symmetrical object and in Fig.7 for the grayscale texture.

We found that the visual servo converged in both the parallel and the non parallel configurations (Figs. 6c, 6d). An exponential decrease of the errors and velocities were obtained, even though only $\mathbf{L}_{\mathbf{s} *}$ was used in the control scheme. With the symmetrical object with parallel desired pose (See Fig.6a), let us note that the target leaves the camera field of view for a brief time period but the visual servoing converges eventually to the right values (this can be seen clearly in the videos).

The second set of experiments were performed with photometric image moments. The behaviour was very satisfactory in terms of convergence and exponential decrease

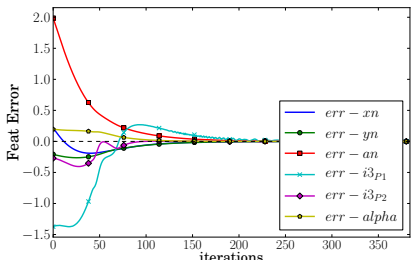

(a)

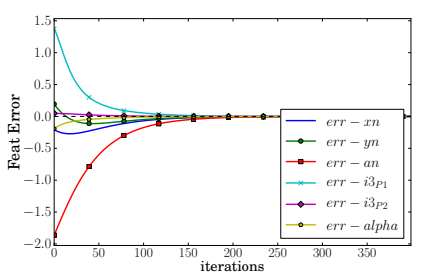

(c)

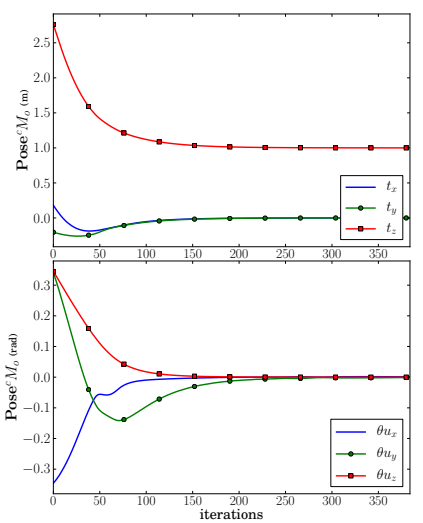

(e)

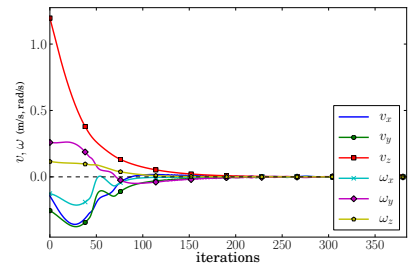

(b)

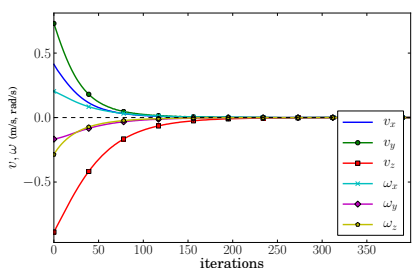

(d)

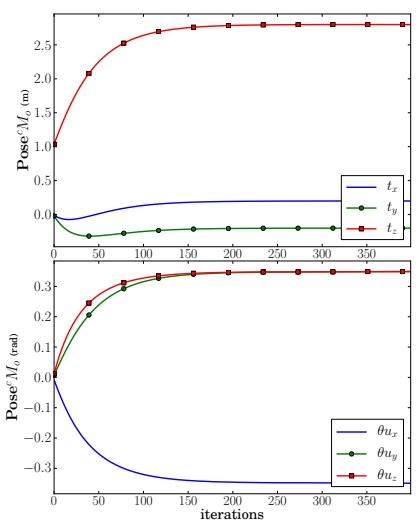

(f)
Fig. 6: Servoing results for Case III-A -Symmetrical Object (a) Errors in visual features, (b) Camera velocities for the parallel desired configuration, (c) Errors in visual features, (d) Camera velocities for the non-parallel desired configuration, and (e) and (f) Robot pose for the parallel and non-parallel configurations respectively.

of the feature errors. The method clearly works irrespective of parallel or non-parallel configurations. The servo results are shown in Fig.7. An important point to be stressed here is that all these results were obtained using only the interaction matrix at the desired position $\mathbf{L}_{\mathbf{s} *}$. Thus, with the optimal values of tunable shift points and visual features selected as selected above, we can design control schemes that results in adequate servo characteristics.

\section{CONClusions ANd Future Work}

We introduced the concept of tunable shift points and proposed various metrics that can be used to tune them for optimal behavior. Visual features based on moment invariants are then computed from these optimally tuned shift points. The visual features built from these moment invariants are used to control the $x$ and $y$ rotations in a 6-DOF VS control scheme. In short, the 'tunable' property of shift points was exploited to solve the important problem of controlling 


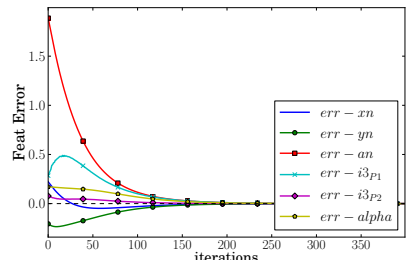

(a)

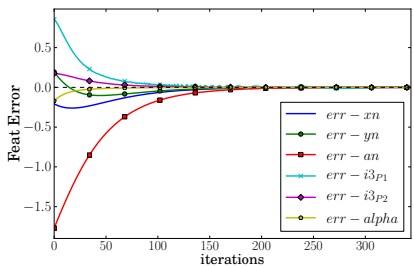

(c)

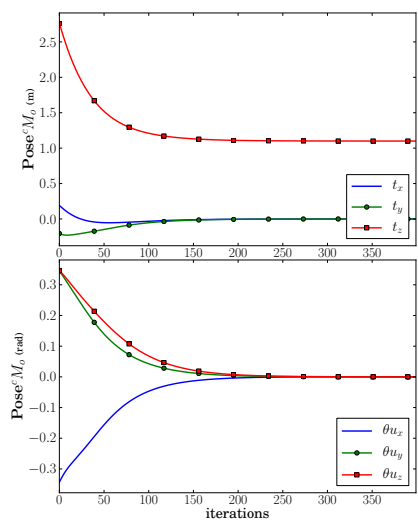

(e)

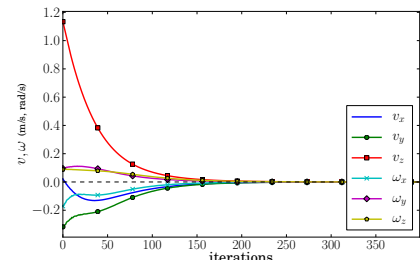

(b)

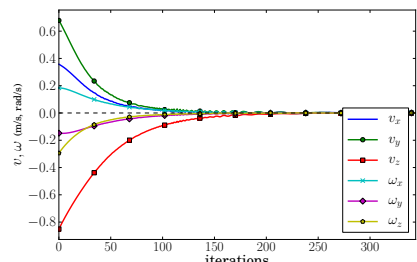

(d)

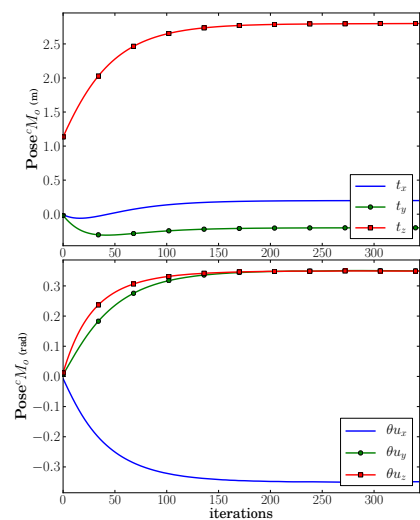

(f)
Fig. 7: Servoing results for Case III-B - Photometric target (a) Errors in visual features, (b) Camera velocities for the parallel desired configuration, (c) Errors in visual features, (d) Camera velocities for the non-parallel desired configuration, and (e) and (f) Robot pose for the parallel and non-parallel configurations respectively.

rotational motions around the the $x$ and $y$ camera axes. The proposed methods and ideas were first validated in simulation with a simple symmetrical object and then with a photometric target. A real experiment conducted on a 6-DOF gantry robot using photometric moments has been presented in the accompanying video.

The proposed method of customizing the shift points is not tied to any specific visual feature. Any moment invariant based visual feature can benefit from this method. This work has opened a new perspective with regards to stability of moments-based visual servoing schemes, that is yet to be demonstrated. As future work, we would also like to develop analytical methods to characterize the behaviour of $\Delta_{1}$ and $\Delta_{2}$. We would also like to explore if this methodology could be adapted to have specific desired characteristics during the visual servoing like more decoupling in the control or a large convergence domain (which is directly related to stability).

\section{REFERENCES}

[1] F. Chaumette and S. Hutchinson, "Visual servo control, part i: Basic approaches," IEEE Robot. Autom. Mag., vol. 13, no. 4, pp. 82-90, Dec. 2006.

[2] C. Collewet, E. Marchand, and F. Chaumette, "Visual servoing set free from image processing," in IEEE Int. Conf. on Robotics and Automation, ICRA'08, Pasadena, California, May 2008, pp. 81-86.

[3] R. Fomena, C. Perez Quintero, M. Gridseth, and M. Jagersand, "Towards practical visual servoing in robotics," in Computer and Robot Vision (CRV), 2013 International Conference on, 2013, pp. 303310 .

[4] E. Malis, F. Chaumette, and S. Boudet, "2-1/2d visual servoing," Robotics and Automation, IEEE Transactions on, vol. 15, no. 2, pp. 238-250, Apr.

[5] C. Collewet and E. Marchand, "Photometric visual servoing," IEEE Trans. on Robotics, vol. 27, no. 4, pp. 828-834, Aug. 2011.

[6] A. Dame and E. Marchand, "Mutual information based visual servoing," IEEE Trans. on Robotics, vol. 27, no. 5, pp. 958-969, Oct. 2011.

[7] F. Chaumette, "Image moments: a general and useful set of features for visual servoing," IEEE Trans. on Robotics, vol. 20, no. 4, pp. 713-723, Aug. 2004.

[8] O. Tahri and F. Chaumette, "Point-based and region-based image moments for visual servoing of planar objects," IEEE Trans. on Robotics, vol. 21, no. 6, pp. 1116-1127, Dec. 2005.

[9] G. Wells, C. Venaille, and C. Torras, "Vision-based robot positioning using neural networks," Image and Vision Computing, vol. 14(10), pp. 715-732, 1996.

[10] J. Wang and H. Cho, "Micropeg and hole alignment using image moments based visual servoing method," IEEE Trans. on Industrial Electronics, vol. 55(3), pp. 1286-1294, 2008.

[11] M. Bakthavatchalam, F. Chaumette, and E. Marchand, "Photometric moments: New promising candidates for visual servoing," in IEEE Int. Conf. on Robotics and Automation, ICRA'13, Karlsruhe, Germany, May 2013, pp. 5521-5526.

[12] V. Kallem, M. Dewan, J. Swensen, G. Hager, and N. Cowan, "Kernelbased visual servoing," in IEEE/RSJ Int. Conf. on Intelligent Robots and Systems, IROS'07. San Diego, California: IEEE, Nov. 2007, pp. 1975-1980.

[13] A. Yeremou Tamtsia, O. Tahri, Y. Mezouar, H. Djalo, and T. Tonye, "New results in image moments-based visual servoing," in IEEE International Conference on Robotics and Automation 2013, Karlsruhe, Germany, May 2013.

[14] C. H. Teh and R. T. Chin, "On image analysis by the method of moments," vol. 10, no. 4, pp. 496-513, 1988. [Online]. Available: http://ieeexplore.ieee.org/xpls/abs_all.jsp?arnumber=3913

[15] J. Feddema, C. S. G. Lee, and O. Mitchell, "Weighted selection of image features for resolved rate visual feedback control," Robotics and Automation, IEEE Transactions on, vol. 7, no. 1, pp. 31-47, Feb 1991.

[16] B. J. Nelson and P. K. Khosla, "Strategies for increasing the tracking region of an eye-in-hand system by singularity and joint limit avoidance," The International journal of robotics research, vol. 14, no. 3, pp. 255-269, 1995.

[17] R. Sharma and S. Hutchinson, "Motion perceptibility and its application to active vision-based servo control," Robotics and Automation, IEEE Transactions on, vol. 13, no. 4, pp. 607-617, 1997.

[18] E. Marchand, F. Spindler, and F. Chaumette, "ViSP for visual servoing: a generic software platform with a wide class of robot control skills," IEEE Robot. Autom. Mag., vol. 12, no. 4, pp. 40-52, Dec. 2005. 